\title{
Interpretation of multi-detector computed tomography images before dissection may allow detection of vascular anomalies: a postmortem study of anomalous origin of the right subclavian artery and the right vertebral artery
}

\author{
Noboru Sakamoto $\cdot$ Hidenobu Miyaso $\cdot$ Masatoshi Komiyama Yota Sugata \\ Takane Suzuki • Toshihiko Kohno • Hirotaro Iwase • Mutsumi Hayakawa • \\ Go Inokuchi · Chisato Mori · Yoshiharu Matsuno
}

Received: 20 December 2011/Accepted: 9 August 2012/Published online: 29 August 2012

(C) The Author(s) 2012. This article is published with open access at Springerlink.com

\begin{abstract}
The Graduate School of Medicine at Chiba University is planning to introduce computed tomography (CT) images of donated cadavers to the gross anatomy laboratory. Here we describe an anomaly of the right subclavian artery that was detected by interpretation of $\mathrm{CT}$ images prior to dissection. The anomaly was verified to be the right subclavian artery, as the last branch of the aortic arch, by subsequent dissection of the cadaver. We also identified an anomalous origin of the right vertebral artery by dissection. This anomaly was also visible on CT images, although it had not been recognized in the first interpretation of the CT images. Our results suggest that branching anomalies of arteries with a diameter of $>1 \mathrm{~cm}$ are detectable on CT images even without the injection of contrast medium. We also discuss the utility of interpreting $\mathrm{CT}$ images prior to dissection as a means by which medical students can gain a better understanding of human body during the gross anatomy laboratory.
\end{abstract}

Keywords Computed tomography · Variation ·

Subclavian artery $\cdot$ Vertebral artery $\cdot$ Anatomical education

\section{Introduction}

In recent years the interpretation of computed tomography (CT) images has become indispensable for the diagnosis of

N. Sakamoto $\cdot$ H. Miyaso - M. Komiyama · Y. Sugata ·

T. Suzuki · T. Kohno · C. Mori · Y. Matsuno $(\bowtie)$

Department of Bioenvironmental Medicine,

Graduate School of Medicine, Chiba University,

Inohana 1-8-1, Chuo-ku, Chiba 260-8670, Japan

e-mail: ymatsuno@faculty.chiba-u.jp

H. Iwase $\cdot$ M. Hayakawa · G. Inokuchi

Department of Legal Medicine, Graduate School of Medicine,

Chiba University, Chiba, Japan diseases and the evaluation of treatment in the clinical setting. It is also possible that anatomical anomalies in patients may also be identified when $\mathrm{CT}$ images are being interpreted (Goray et al. 2005; Ka-Tak et al. 2007; Park et al. 2008). It has also been reported that partial resection of the pectoralis major muscle in association with surgery for breast cancer and anomalous shape of the vertebral column with the lateral curvature can be recognized prior to dissection based on the interpretation of CT images of cadavers (Matsuno et al. 2009). Thus, interpretation of CT images may be helpful not only in the clinical setting but also in gross anatomy laboratory. In fact, the use of CT images in anatomical education classes has already been initiated in Japan (Murakami et al. 2011). The Graduate School of Medicine, Chiba University, is also planning to introduce $\mathrm{CT}$ images of donated cadavers to its gross anatomy laboratory. As such, medical students can dissect cadavers after viewing CT images of their dissecting cadavers that were obtained in advance.

Here, we describe a cadaver in which we detected an anomaly of the right subclavian artery by interpreting CT images prior to dissection. We verified the anomaly by subsequent dissection of the cadaver and also identified an anomalous origin of the right vertebral artery that had not been recognized on the $\mathrm{CT}$ images. We also discuss the utility of interpreting CT images before dissection for identifying vascular anomalies and for gaining a better understanding of the human body by students in the gross anatomy laboratory.

\section{Methods \\ Conducting CT scans of cadavers}

At Chiba University, consent for cadaver use for the purpose of medical education, anatomy studies, and CT scans is 
obtained from members of the whole body donation registry and their families. CT scans are performed on cadavers only after such consent has been obtained. All CT scans of cadavers have been approved by the ethics committee of Graduate School of Medicine, Chiba University (No. 521).

CT scan of the cadaver and settings for CT image interpretation

The cadaver used in this study was an 84-year-old Japanese woman who died of lung cancer. After the cadaver had been embalmed and fixed by perfusion with a solution containing formaldehyde, a CT scan was performed. No contrast medium was injected.

The whole cadaver was scanned at a $1.25-\mathrm{mm}$ slice interval using an ECLOS 16-line Multi-detector Computed Tomography (Hitachi Medical Co., Tokyo, Japan). Scanning parameters were $120 \mathrm{kV}$ and $200 \mathrm{~mA}$ for the tube voltage, and $1.25 \times 16 \mathrm{~mm}$ for the beam collimation. CT images were reconstructed at a section thickness of $1.25 \mathrm{~mm}$. The software used to view CT image data was OsiriX MD ver. 1.0 64-bit.

\section{Results}

Interpretation of $\mathrm{CT}$ images before dissection

Interpretation of the CT images before dissection revealed a branching anomaly of the aortic arch (Fig. 1a-d). In a horizontal section at the level of the third thoracic vertebra, an unusual structure that appeared to be an artery was detected passing behind the esophagus (Fig. 1a). This structure seemed to branch from the aortic arch. When it was followed upward, it was found to be located on the right side of the esophagus at the level of the boundary between the second and third thoracic vertebrae (Fig. 1b) and then turned to the right at the level of the second thoracic vertebra (Fig. 1c). Three arteries were observed in front of the trachea (Fig. 1a, b). One of these ascended along the right side of the trachea without branching off the right subclavian artery (Fig. 1b, c) and thus appeared not to be the brachiocephalic artery, but the right common carotid artery. The other two on the left side of the trachea ascended taking normal courses and seemed to be the left common carotid artery and the left subclavian artery, respectively (Fig. 1b, c). Thus, the artery-like structure passing behind the esophagus seemed to be the right subclavian artery.

Assessment based on dissection of the area of malformation detected on CT images

The dissection conducted to assess the branching anomaly of the aortic arch detected on the CT images confirmed malformation of the right subclavian artery, i.e. as the last branch of the aortic arch (Figs. 2,3). The right subclavian
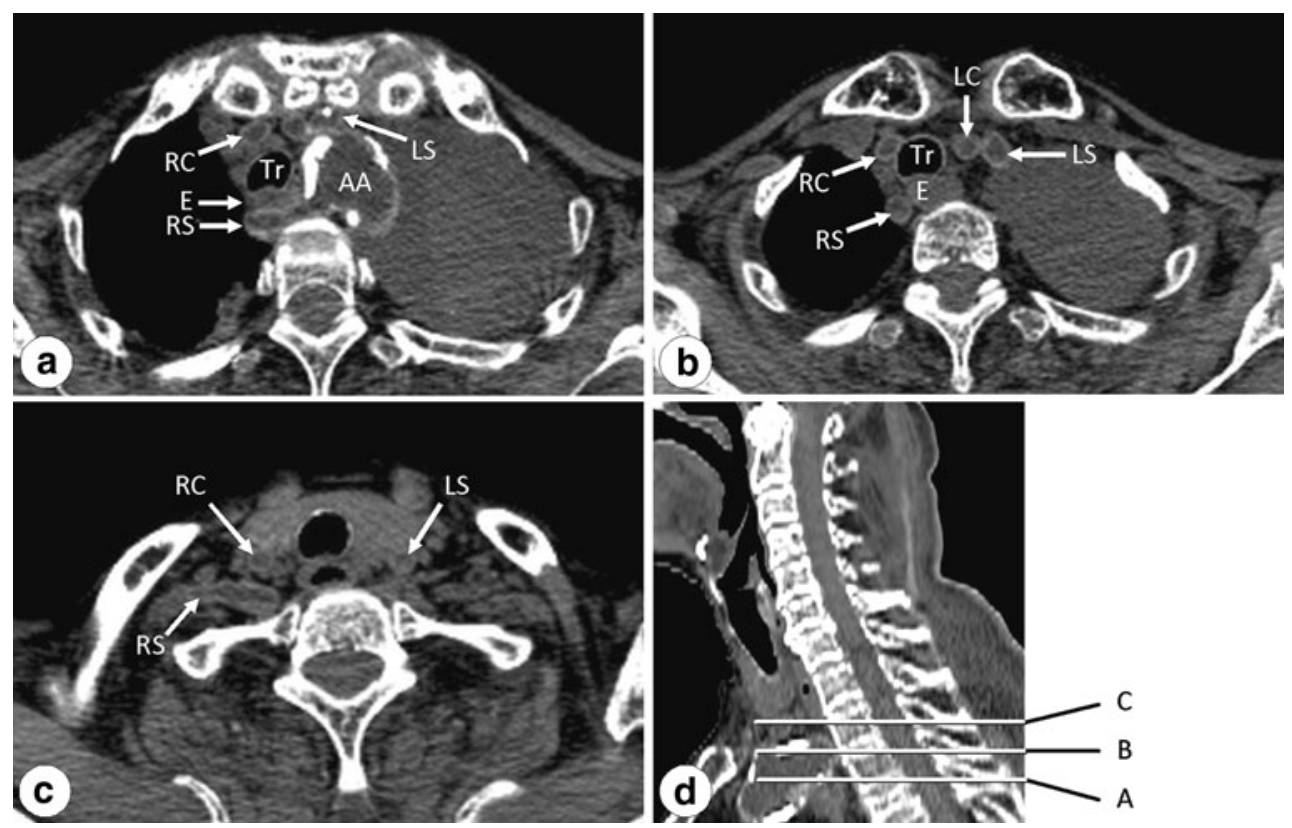

Fig. 1 Computed tomograph images of horizontal sections at the level of the third thoracic vertebra (a), at the boundary between the second and third thoracic vertebrae (b), and at the second thoracic vertebra (c). d A sagittal section indicating the horizontal section levels of CT images in $\mathbf{a}, \mathbf{b}$, and $\mathbf{c}(A, B$, and $C$, respectively).

The right subclavian artery can be confirmed to be crossing between the vertebral body and esophagus (a). $A A$ Aortic arch, $E$ esophagus, $L C$ left common carotid artery, $L S$ left subclavian artery, $R C$ right common carotid artery, $R S$ right subclavian artery, $\operatorname{Tr}$ trachea 

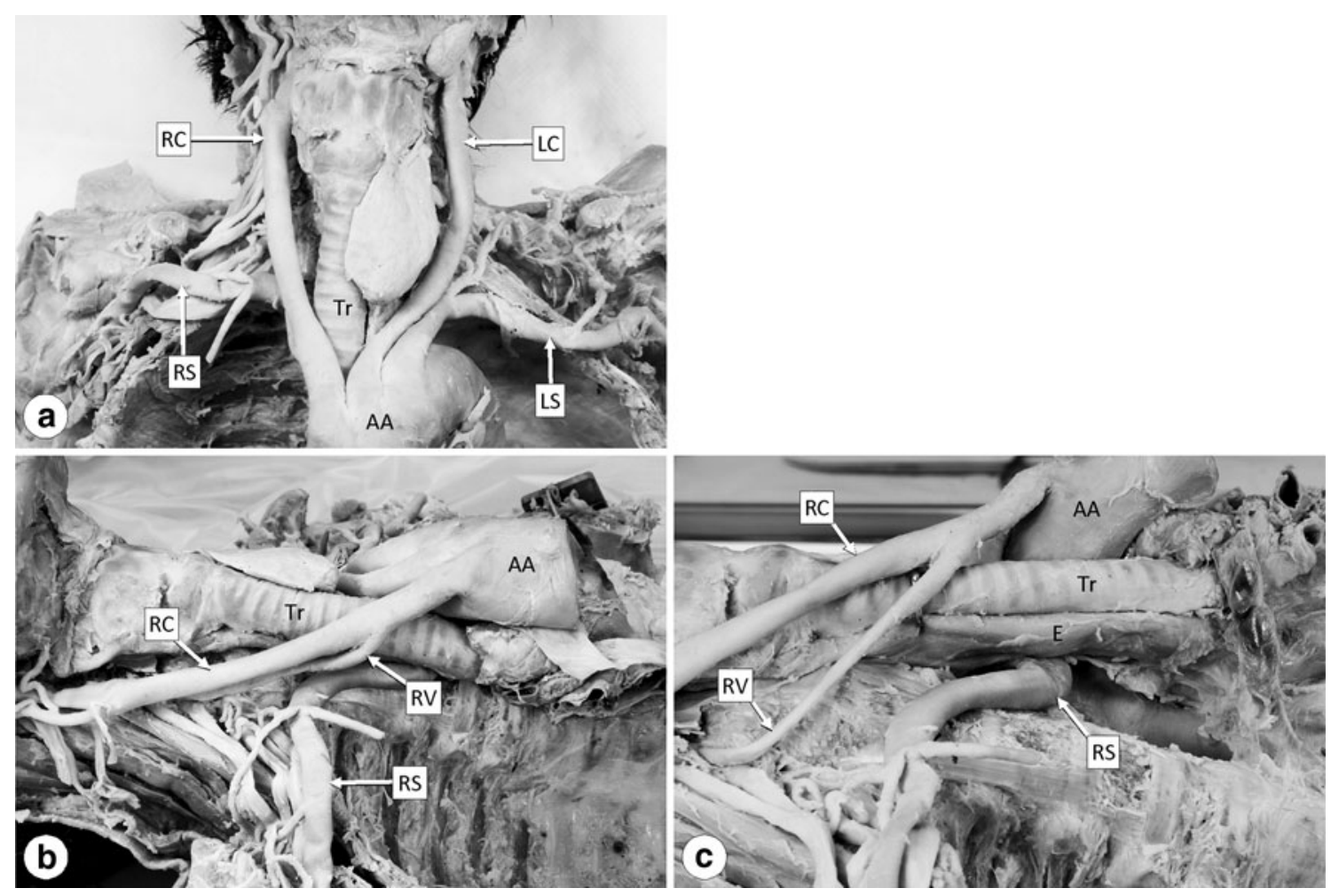

Fig. 2 Photographs showing the anterior $(\mathbf{a})$ and right side view $(\mathbf{b}, \mathbf{c})$ of the arteries branching from the aortic arch $(A A)$. $\mathbf{c}$ The AA is pulled towards the front to show the right vertebral artery $(R V)$ and esophagus $(E)$. All abbreviations are as given in Fig. 1
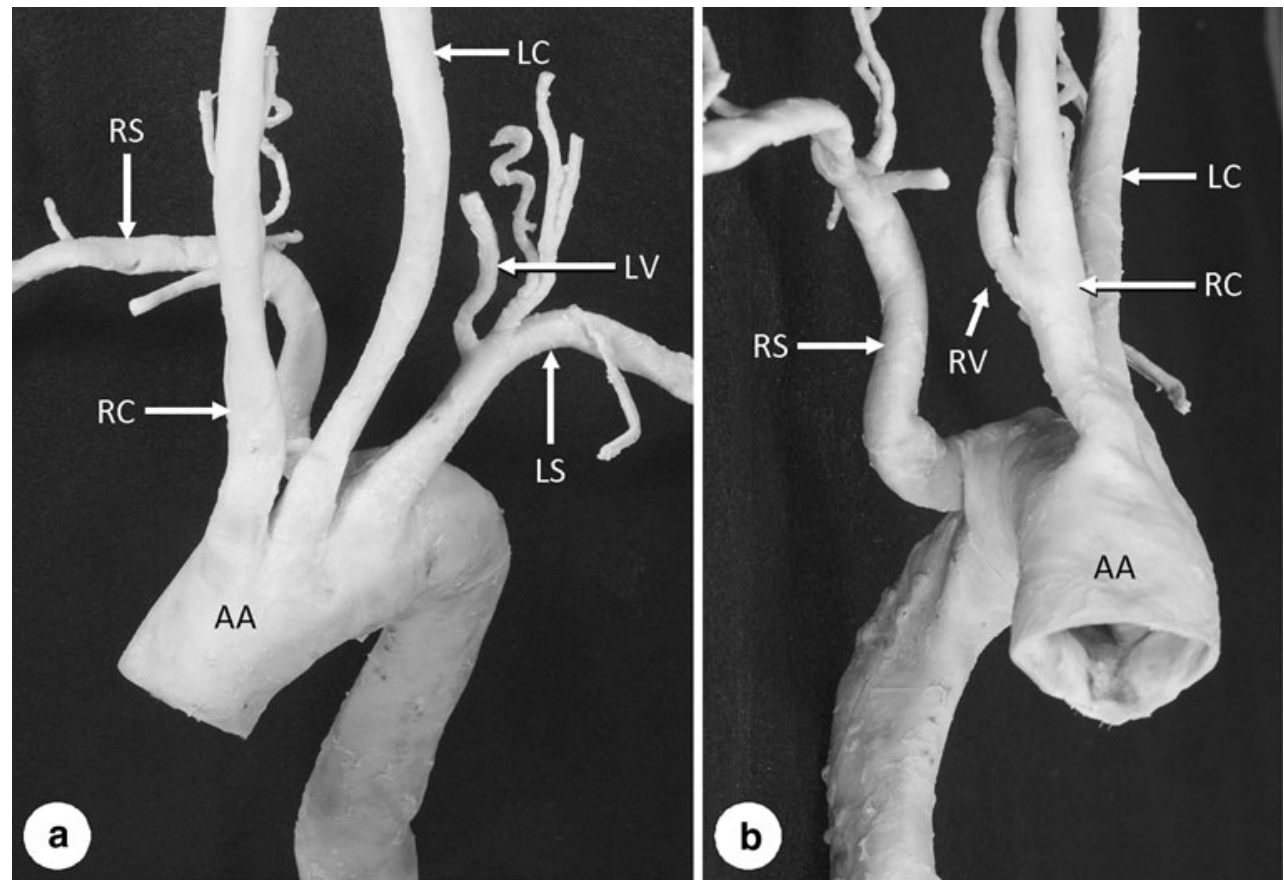

Fig. 3 Photographs showing the anterior (a) and right side view (b) of the aortic arch $(A A)$ and its branches. All abbreviations are as given in Fig. 1

artery branched from the medial posterior surface of the aortic arch as its last branch at the level of the second and third thoracic vertebra (Fig. 3). It coursed obliquely to the upper right between the posterior surface of the esophagus and the anterior surface of the second and third thoracic vertebral bodies and then ascended (Fig. 2b, c) and crossed 
rightward to become the axillary artery at the level of the first thoracic vertebra (Fig. 2a).

The right inferior laryngeal nerve branched directly from the right vagus nerve in the cervical region, and thus the right recurrent laryngeal nerve was absent. In contrast, the left recurrent laryngeal nerve was normal. The ligamentum arteriosum was also normal.

In addition to the above-described anomalous origin of the right subclavian artery, it was also revealed that the right vertebral artery branched from the right common carotid artery and entered the transverse foramen of the fifth cervical vertebra (Figs. 2b, c, 3b). In contrast, the left vertebral artery branched from the left subclavian artery (Fig. 3a) and ascended through the transverse foramen from the sixth to the first cervical vertebra, thus showing the normal form.

Table 1 shows the diameters (major axis/minor axis) of the arteries branching from the aortic arch. The diameters were measured just after branching (at approximately $5 \mathrm{~mm}$ from the branching site). The major axis of the right subclavian artery had a diameter of $13.2 \mathrm{~mm}$ and its minor axis a diameter of $4.5 \mathrm{~mm}$; The right subclavian artery also had a flattened appearance from its origin up to the location on the right side of the esophagus (Fig. 3b). This appearance might have been caused by compression because the right subclavian artery coursed obliquely to the upper right between the esophagus and thoracic vertebral bodies. However, it is unclear whether the compression occurred during lifetime or after death. The esophagus showed only slight compression, possibly due to the right subclavian artery, and was also flat (Fig. 2c).

The major axis of the right vertebral artery had a diameter of $5.5 \mathrm{~mm}$ and the minor axis a diameter of $4.6 \mathrm{~mm}$ (Table 1). Both diameters were larger than those of the left vertebral artery (major axis $4.5 \mathrm{~mm}$, minor axis $3.6 \mathrm{~mm})$.

\section{Review of CT images after dissection}

Although the interpretation of CT images before dissection revealed the aberrant right subclavian artery, the anomaly of the right vertebral artery was not identified at this time. When the anomalous origin and course of the right vertebral artery detected by dissection were checked again on the CT images, it could be confirmed on the horizontal and sagittal section images at the level of the fifth cervical vertebra that this artery enters the right transverse foramen of the fifth cervical vertebra (Fig. 4). However, the branching site from the right common carotid artery could not be identified due to insufficient image clarity.
Table 1 Diameters (major axis/minor axis) of the arteries branching from the aortic arch

\begin{tabular}{lll}
\hline Arteries & $\begin{array}{l}\text { Major axis } \\
(\mathrm{mm})\end{array}$ & $\begin{array}{l}\text { Minor axis } \\
(\mathrm{mm})\end{array}$ \\
\hline $\begin{array}{l}\text { Subclavian artery } \\
\quad \text { Right }\end{array}$ & $13.2^{\mathrm{a}}$ & 4.5 \\
$\quad$ Left & 11.0 & 7.8 \\
Common carotid artery & 11.7 & 7.9 \\
$\quad$ Right & 9.9 & 7.9 \\
$\quad$ Left & & \\
Vertebral artery & 5.5 & 4.6 \\
$\quad$ Right & 4.5 & 3.6 \\
$\quad$ Left & & \\
Aortic arch & \\
$\quad \begin{array}{l}\text { Proximal to the branching site of the } \\
\text { right subclavian artery }\end{array}$ & 32.0 & 27.0 \\
$\quad \begin{array}{l}\text { Distal to the branching site of the right } \\
\text { subclavian artery }\end{array}$ & 33.2 & 23.3 \\
\hline
\end{tabular}

a Each diameter was measured at approximately $5 \mathrm{~mm}$ from the branching site

What organs can be interpreted on CT images without contrast medium?

Even without contrast medium, bones and calcified deposits along arteries were easy to interpret on the CT images of the cadaver. Also, major organs (heart, lung, liver, kidney, brain, spinal cord, major muscles like biceps brachii muscle and gluteus maximus muscle, etc.) were recognizable, but the interpretation of veins, peripheral nerves, and smaller muscles were difficult.

\section{Discussion}

Anomalous origin of the right subclavian artery and the right vertebral artery

The branching pattern of the aortic arch in the cadaver under discussion resembled that of Type $\mathrm{G}$ or Type CG of the Adachi-Williams-Nakagawa classification (Adachi 1928; Williams et al. 1932; Nakagawa 1939), in which both types have a retroesophageal right subclavian artery and anomalous origin of the vertebral arteries. However, the left vertebral artery in our study was normal, although anomalous origin of the left vertebral artery is also involved in both Type $\mathrm{G}$ and Type CG. Thus, classification of the branching pattern is slightly difficult in this cadaver.

Normally, the right subclavian artery is formed with the seventh intersegmental artery, which arises from the right dorsal aorta, after obliteration of the peripheral segment of 


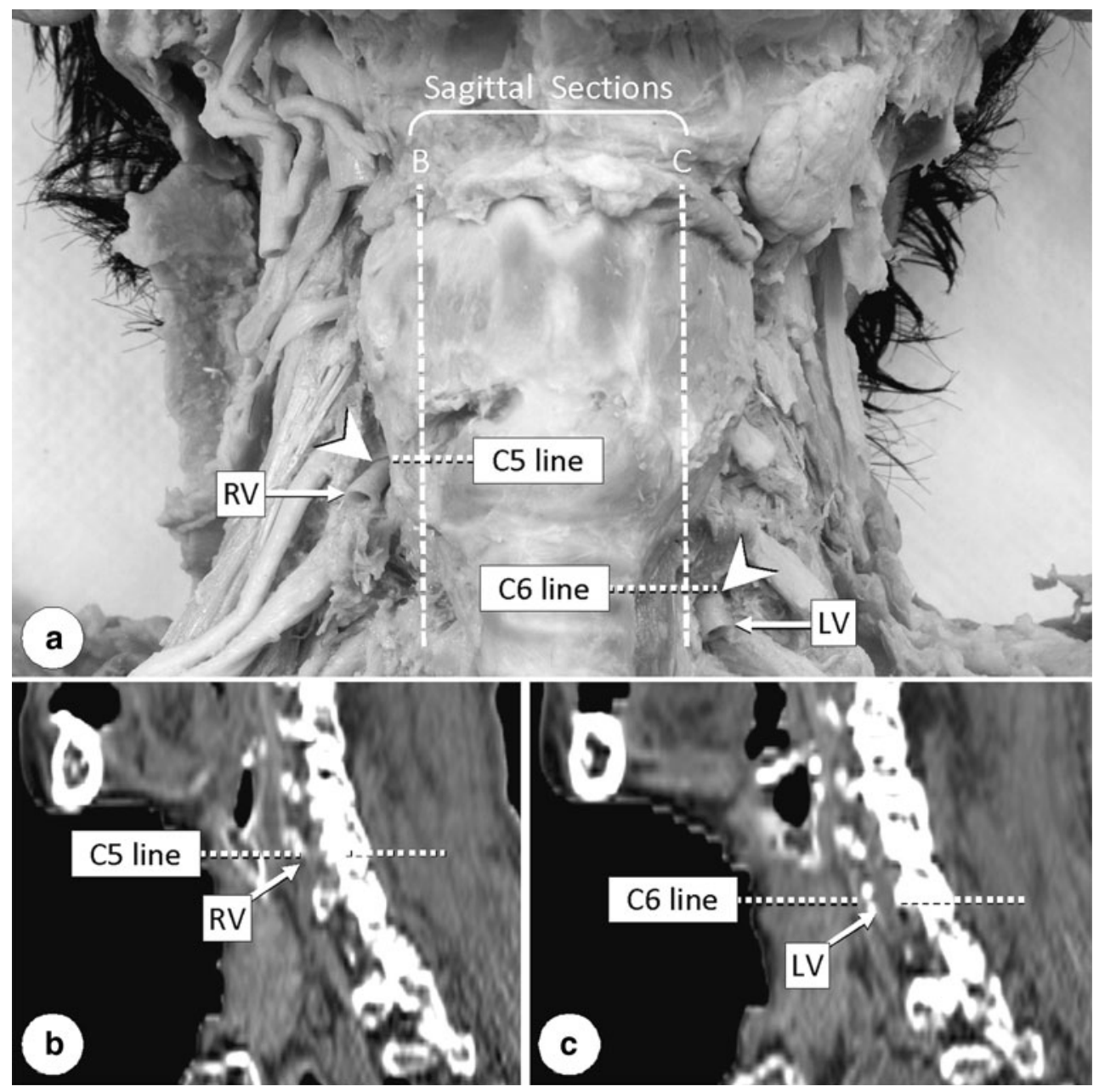

Fig. 4 Anterior view (a) and sagittal sections (b, c) showing vertebral arteries entering the transverse foramina. Arrowheads (a) indicate the entering sites. Vertical lines $(B$ and $C$ ) in a indicate

the right dorsal aorta (Moore 1982; Wasserman et al. 1992; Lemke et al. 1999). If the obliteration occurs proximally to the seventh intersegmental artery, the right subclavian artery starts to branch from the aortic arch as its last branch (Wasserman et al. 1992; Lemke et al. 1999). It has been reported that the right recurrent laryngeal nerve is usually absent when the right subclavian artery branches from the aortic arch and takes a retroesophageal course (Kawashima and Sasaki 2005). In our study, the right recurrent laryngeal nerve was also absent from the cadaver. The vertebral artery is formed by the development of longitudinal anastomoses between the cervical (from the first to the seventh) intersegmental arteries, and thus the vertebral artery normally arises from the subclavian artery and enters the transverse foramen of the sixth cervical vertebra (Lemke et al. 1999; Ikegami et al. 2007; Park et al. 2008). In our study, the segment of the right dorsal aorta and the longitudinal anastmosis between the sixth and seventh intersegmental arteries might have regressed and, instead, the the positions of sagittal sections in $\mathbf{b}$ and $\mathbf{c}$, respectively. $C 5$ Fifth cervical vertebra, $C 6$ sixth cervical vertebra, $L V$ left vertebral artery, $R V$ right vertebral artery

peripheral segment of the right dorsal aorta and the proximal part of the sixth intersegmental artery might have survived. If this were to be the case, the proximal part of the right vertebral artery in this cadaver was formed with the fourth aortic arch, a part of the dorsal aorta and proximal part of the sixth intersegmental artery. This may be related with the fact that the diameter of the right vertebral artery is larger than that of the left vertebral artery in this cadaver.

The incidence of the right subclavian artery as the last branch of the aortic arch has been reported in Japanese adults as ranging from 0.15 to $1.6 \%$ with an average of about $0.5 \%$ (Komiyama et al. 1995). With respect to the vertebral artery, the left one has been reported to show anomalous origin at a frequency of $2.4-5.9 \%$ in several large autopsy series (Lemke et al. 1999; Akdeniz et al. 2007; Ikegami et al. 2007). However, an anomalous origin of the right vertebral artery has been reported to be rarer, being detected as a coincidental finding during surgery or 
at autopsy (Akdeniz et al. 2007). In fact, many of the reports describing anomalous origin of the right vertebral artery are coincidental findings during surgery (Palmer 1977; Rieger and Huber 1983; Chen et al. 1998; Lemke et al. 1999; Best and Bumpers 2002; Yanik et al. 2004; Goray et al. 2005; Akdeniz et al. 2007; Ka-Tak et al. 2007; Satti et al. 2007; Kau et al. 2008; Park et al. 2008; Kim et al. 2009), although such findings at autopsy have also been reported (Mori et al. 1990; Gluncic et al. 1999; Fazan et al. 2004; Ikegami et al. 2007). The same concomitant anomalies of the right subclavian artery and right vertebral artery as those observed in our study have been reported previously, although the vertebral artery enters the transverse foramen at various levels (Mori et al. 1990; Gluncic et al. 1999; Fazan et al. 2004; Ka-Tak et al. 2007; Kau et al. 2008; Park et al. 2008; Kim et al. 2009). Consequently, the findings we report here may be not be that exceptional.

Possibility of detecting anomalies on CT images before dissection

We detected branching anomaly of the subclavian artery on CT images examined prior to dissection. At that time, however, anomaly of the vertebral artery could not be detected. The detection-or not—of these anomalies may depend on the size and thickness of the wall of arteries, which affect the visibility of arteries on CT images. The subclavian artery is approximately $1 \mathrm{~cm}$ in diameter and considered to be an elastic type artery which has a thicker wall. In comparison, the vertebral artery is usually $4-5 \mathrm{~mm}$ in diameter and might be a muscular type artery with a thinner wall. Our results suggest that branching anomalies of arteries with a diameter of $>1 \mathrm{~cm}$ are detectable on CT images even without the injection of contrast medium. The diagnosis of anomalies of thinner arteries that are several millimeters in diameter may also be possible depending on the techniques applied to interpret the CT images. The use of contrast medium definitely makes the diagnosis easier, as has been shown in previous reports (Palmer 1977; Rieger and Huber 1983; Chen et al. 1998; Lemke et al. 1999; Best and Bumpers 2002; Yanik et al. 2004; Goray et al. 2005; Akdeniz et al. 2007; Ka-Tak et al. 2007; Satti et al. 2007; Kau et al. 2008; Park et al. 2008; Kim et al. 2009; Matsuno et al. 2009).

Various anomalies are usually encountered in the gross anatomy laboratory. However, we believe that there may be numerous cadavers in which these anomalies go undetected in the gross anatomy laboratory because medical students who are just learning normal structure are not yet skilled in differentiating anomalies from normal structure. If the presence of an anomaly is known prior to dissection, students can pay attention to the anomaly and dissect the cadaver carefully to find the anomaly. The interpretation of
CT images prior to dissection may therefore be a useful educational tool for medical students to detect anomalies during the gross anatomy laboratory.

For a better understanding of human body structure by medical students

Computed tomography images are frequently used in the clinical setting for the diagnosis of diseases and evaluation of treatments. In order to interpret CT images, it is important for the physician to be able to correlate two-dimensional CT images with the actual three-dimensional structures of the body. Although medical students can observe inner structures of the human body three-dimensionally in the gross anatomy laboratory, they sometimes lack knowledge of the two-dimensional aspects of the structures during the actual dissection. Clinical trainees also have few opportunities to relearn inner structures of the whole body three-dimensionally by dissection, even though they have many opportunities to view two-dimensional CT images. Thus, when dissecting a cadaver, medical students may find it easier to correlate CT images with the real structure if they have had the opportunity to view CT images of that cadaver prior to the dissection. Thus, CT scanning of donated cadavers and the introduction of CT images of the cadavers to gross anatomy laboratories may be a very useful educational approach that provides medical students with a better understanding of human body structure. If clear threedimensional reconstructed images can be obtained from cadavers, such images would clearly be useful for anatomical education. However, it is difficult to obtain clear threedimensional images at the present time due to insufficient contrast between organs. Thus, improved methods that allow optimal imaging of CT specimens are urgently needed.

Acknowledgments We wish to thank the members of the Chiba Shiragiku community who donated their bodies to science. This work was supported by JSPS KAKENHI Grant Number 22590169.

\section{Conflict of interest None.}

Open Access This article is distributed under the terms of the Creative Commons Attribution License which permits any use, distribution, and reproduction in any medium, provided the original author(s) and the source are credited.

\section{References}

Adachi B (1928) Das Arteriensystem der Japaner. Bd I. KaiserlichJapanischen Universität zu Kyoto, Kyoto, pp 22-41

Akdeniz B, Yilmaz E, Pekel N et al (2007) Anomalous origin of the right vertebral artery from the ascending aorta in the presence of an aberrant right subclavian artery. Int J Cardiovasc Imaging 23:39-42 
Best IM, Bumpers HL (2002) Anomalous origins of the right vertebral, subclavian, and common carotid arteries in a patient with a four-vessel aortic arch. Ann Vasc Surg 16:231-234

Chen CJ, Wang LJ, Wong YC (1998) Abnormal origin of the vertebral artery from the common carotid artery. AJNR Am J Neuroradiol 19:1414-1416

Fazan VPS, Caetano AG, Filho OAR (2004) Anomalous origin and cervical course of the vertebral artery in the presence of a retroesophageal right subclavian artery. Clin Anat 17:354-357

Gluncic V, Ivkic G, Marin D et al (1999) Anomalous origin of both vertebral arteries. Clin Anat 12:281-284

Goray VB, Joshi AR, Garg A et al (2005) Aortic arch variation: a unique case with anomalous origin of both vertebral arteries as additional branches of the aortic arch distal to left subclavian artery. AJNR Am J Neuroradiol 26:93-95

Ikegami A, Ohtani Y, Ohtani O (2007) Bilateral variations of the vertebral arteries: the left originating from the aortic arch and the left and right entering the C5 transverse foramina. Anat Sci Int 82:175-179

Ka-Tak W, Lam WWM, Yu SCH (2007) MDCT of an aberrant right subclavian artery and of bilateral vertebral arteries with anomalous origins. AJR Am J Roentgenol 188:W274-W275

Kau T, Gasser J, Lesnik G et al (2008) Images in vascular medicine. Two exceptionally rare branching patterns of the aortic arch. Vasc Med 13:89-90

Kawashima T, Sasaki H (2005) Topological changes of the human autonomic cardiac nervous system in individuals with a retroesophageal right subclavian artery: two case reports and a brief review. Anat Embryol 210:327-334

Kim YD, Yeo HT, Cho YD (2009) Anomalous variations of the origin and course of vertebral arteries in patients with retroesophageal right subclavian artery. J Korean Neurosurg Soc 45:297-299

Komiyama M, Matsuno Y, Shimada Y (1995) Variation of the right subclavian artery as the last branch of the aortic arch in two Japanese cadavers. Okajimas Folia Anat Jpn 72:245-247

Lemke AJ, Benndorf G, Liebig T et al (1999) Anomalous origin of the right vertebral artery: review of the literature and case report of right vertebral artery origin distal to the left subclavian artery. AJNR Am J Neuroradiol 20:1318-1321
Matsuno Y, Yamamoto S, Miyaso H, Ohta M, Suzuki T, Komiyama M, Mori C (2009) Trial application of computed tomography (CT) to donated cadavers during human gross anatomy laboratories and anticipated educational effects (in Japanese). Chiba Med J 85:237-240

Moore KL (1982) The developing human, 3rd edn. Saunders, Philadelphia

Mori C, Hashimoto H, Hoshino K (1990) Two cases of double superior vena cava. Jpn Heart J 31:881-888

Murakami T, Tajika Y, Ueno $\mathrm{H}$ et al (2011) Integrated course of anatomy and CT radiology for the edge of medicine. J Physiol Sci 61[Supp 1]:S248

Nakagawa M (1939) On the branching pattern of the aortic arch (in Japanese). J Juzen Med Soc 44:243-259

Palmer FJ (1977) Origin of the right vertebral artery from the right common carotid artery: angiographic demonstration of three cases. Br J Radiol 50:185-187

Park JK, Kim SH, Kim BS, et al (2008) Two cases of aberrant right subclavian artery and right vertebral artery that originated from the right common carotid artery. Korean J Radiol 9 [Supp 1]: S39-S42

Rieger P, Huber G (1983) Fenestration and duplicate origin of the left vertebral artery in angiography: report of three cases. Neuroradiology 25:45-50

Satti SR, Cerniglia CA, Koenigsberg RA (2007) Cervical vertebral artery variations: an anatomic study. AJNR Am J Neuroradiol 28:976-980

Wasserman BA, Mikulis DJ, Manzione JV (1992) Origin of the right vertebral artery from the left side of the aortic arch proximal to the orgin of the left subclavian artery. AJNR Am J Neuroradiol 13:355-358

Williams GD, Affe HM, Scheckbier M et al (1932) Variations in the arrangement of the branches arising from the aortic arch in American whites and negroes. Anat Rec 54:247-251

Yanik B, Conkbayir I, Keyik B et al (2004) A rare anomalous origin of right vertebral artery: findings on Doppler sonography. J Clin Ultrasound 32:211-214 